\title{
Redesigning Life with New Science: The Fine Line
}

\author{
Fariel Shafee \\ LLB, University of London, 2020
}

\begin{abstract}
We look into specific aspects of human civilisation the depend on inter-connectivity among related and unrelated individuals. The dependence may be need based or of more fundamental biological nature. Hence the cohesive factors may be rational reciprocity, trust or more instinctive attributes such as loyalty and faith. The biological dependence of members of a complex social structure as well as possible artifacts of local needs and needs of specific periods contribute to the structure the social network as individuals and the environment interact. Hence, stratification is created that are either fundamental or superficial. We analyse the possibilities of shifting the balance of average traditional human behavioural equilibria by introducing modern technologies especially in the fields of enhancement and reproduction. We imagine extreme scenarios to and surmise possible compensations for modern adaptation in behaviour by legal and social means given the uncertainties and complexities of artificially disturbing delicate biological interconnections.
\end{abstract}

Keywords: reproductive ethics, medical jurisprudence, enhancement, artificial reproduction, genetics and society

\section{INTRODUCTION}

$\mathrm{H}$ uman societies are composed of individual members and also of clusters and subgroups that are held together by adhesive factors that may involve needs, sympathy, loyalty and rational decision making to collaborate. However, while each individual has a life to live on his or her own, the members are connected horizontally in a constant time slice within a society where they live together and vertically in between time slices by individuals of different generations. Biologically, human beings are composed of two broad sexes that depend on each other to reproduce though different sexual patterns are also observed in some members. However, recent biotechnological developments offer individuals the possibility of the freedom to have children who are modified or who can be conceived by using genetic material from only one person or even others of the same sex.

In this paper, we first analyse the relationships between one's individual identity and social and biological connections to see if complete autonomy is possible. We look into case laws to deduce how the society values individuals and also connections. We discuss how some connections may be relatively stiffly held by biological factors while others may be shifted more arbitrarily. These notions are taken into considerations to understand personal freedom and its limits. Finally, we imagine some extreme scenariosabout possible courses of social dynamics if some these technological advancements are taken advantages of at large scale. We imagine the effects on individuals and also on the interdependence of members. We look into how these technologies may redefine how human individuals identify themselves and associates with other members as well.

\section{THE HUMAN CONNECTION}

In the first part of the paper, we look into concepts about individuals and propagation of one's personhood. We bring in concepts of biology and also look into case laws to understand the balancing point between a person's autonomy and his or her connections with others. We also analyse the conflicting interests between a society's needs to better itself and hence for each member to gain from the contributions from others and the interest of each individual to perpetuate.

While human individuals have autonomy and personal rights and freedom, human civilization is special in that sense that it developed as an aggregation of knowledge and development of customs. Such aggregation was results of interactions with the environment and with others, and also based on history. Not only knowledge, these aggregated interactions created norms and values within social structures. Hence, though human beings are individuals, they identify with various social instruments and persons, some created by current needs or artificially and some emerging naturally based on more fundamental needs that contributed to the development of such an aggregated structure.

\section{A.Evolution and Generations}

Human beings are said to be genetically 99.9 percent similar to one another if gene identities are considered and 99 percent identical when repeated sequences are taken into account[8]. Yet, ties with families, and needs for families are not always easily replaced with something vast and more generic. Plato [22] imagined that in his perfect city, the most able class will not have families and will share all their belongings instead. This was a theoretical model where the ablest were to undergo their possessionsand ties with immediate kins and would forgo exclusive sexual relations. In practice, a commune based on sharing of sexual partners was experimented with in the Oneida community [6] which strived to create a perfect religion where the children were taken care of by the community as a whole. The experiment did not last long.

Reference [10] discusses a definition of genetic parenthood, and in the wake of modern biotechnology,[2] argues how the definition of genetic relationship may become puzzling when several genomes are spliced together into a baby, when genes are edited etc. Reference [2] casts doubt on how the proportion of genes required to claim parenthood maybe a vague and an ill-defined number. Reference [2] also discusses the case when a person orders a designer baby that is 
engineered for him but is only randomly genetically connected with his own genome. Reference [2] also raises questions about what the notion of derivation of one's gene might imply when genes can be synthetically reproduced using a blue print.

In this paper, by genetic parenthood, we mostly concentrate on the traditional definition where a male and a female offer a sperm and an egg with each carrying fifty percent of the donor's total DNA. We discuss the possible new scenarios as future possibilities of creation of children and try to understand what types of effects the new genetic ordering may have on the society in general. We discuss designer babies, genetic engineering and also a modified version of the scenario where a person can create both a sperm and an ovum. First of all, however, we discuss case laws to understand what, if any, effect, genetic parenthood has on our current socially held values and accepted rights.

\section{B. Biology, Personhood and Extended Identities}

A natural experimentabout the value of genetic parenthood took place when technological development allowed couples or individuals to use others' sperms or eggs to conceive children they wished to be biologically connected with. Though many such children adapted with their situation, stories did emerge where such children looked for their actual biological parents or siblings [9]. They stated that they missed parts of their identity. In light of such situations, the anonymous nature of sperm and egg donation has been modified to allow for children to find for their biological parents as long as the biological parents too consent[9]. When slaves were brought to work in the new land, they were also stripped of their identities and were given new names. Many descendants of such slaves later searched their past identities or identified with their identities based on orally passed down stories[13].

A person does not liveisolated, defined merely by his own attributes, but finds an identity as an amalgamate of institutes, values, historically significant objects and other persons that link up and contribute to building the person's notion of identity. There is a nexus connecting the past to the present and hence weaving an aggregated story that evolves.

Reference [17] has an interesting notion about personhood that results as a product of his interaction with other social institutes. The personhood forms as one discriminates one's own attributes from others' attributes. Reference [17] also discusses the importance of ownership in property as such ownership allows for the fostering of personhood. The raw person is simply capable of attaining such personhood and does attain such as a result of freedom to interact.

Similarly, when a person also leaves behind his property, her heirs are defined largely by family relationships. One does not only support one's young children, and teaches them the ways of life, but also leaves behind his property for the selfsupporting family.
Thus, we see a propagation of property and mores as well as information within close familial connection as well as the existence of public property and schools within a larger community that gives rise to a more extensive sense of fraternal relationships.However, while family ties are closer than relations between strangers within a community, one can sacrifice one's life for one's country or community and in circumstances homicides also occur within a family. Hence, on an average, relationships exist acknowledging deep familial ties within a larger more loosely formed community ties though exceptions do occur and though the strengths of these ties may shift depending on specific needs.

We try to understand the relation between continuity, luck and control by imagining possible social orders. Given that an individual has a finite interval of time associated with his or her existence, what comes before and after is devoid of his exact self's existence but might include other individuals that he or she is continued from physically or might continue into. One has no choice over who one's parents are. That is part of "brute luck" as discussed by Dworkin[11]. One might be born in a rich family and one might be born poor, or in a very underdeveloped part of the world, though one might be, in terms of intelligence and potential, be very similar to another person born elsewhere.

One way to compensate for brute luck and create an egalitarian society would be to take these children under state control and to raise them equally, with equal opportunity, stripping them off of their personal identities. This however, would be contrary to a person's right to have a family. A right to family and privacy is considered to be fundamental in many legal systems, such as in the European Charter for Human Rights (eg.Art 8 HRA 1998, UK).

The other extreme would be to hold children accountable for all their ancestors' sins and levy all their dues from them and to punish them for their ancestors' crimes. However, in most jurisdictions criminal law cases cease to exist when the defendant dies. Some civil suits, however, might survive the death of a defendant. These might include suits regarding pensions, to which the family would be entitled. Any past dues of a defendant are usually levied form the defendant's estate before the estate is divided among heirs. The heirs are not held accountable for debts of a defendant from property earned by themselves.

Whether a person can displace his or her natural heirs form their inheritance rights completely varies among jurisdictions. While in some jurisdictions, one can will one's property to whoever one chooses, in some other jurisdictions one can will only a fraction of one's property[23], hence ensuring that one's heirs are entitled to some parts of his or her remaining property. One however does have ways out in extreme cases. $\mathrm{He}$ or she can sell off one's assets before death or one can create a trust, handing over the legal ownership to another before one's death. 
Hence, it seems that while a respect for autonomy and individual capacities are highly appreciated within the human societies, human beings are in many cases reluctant to be taken away from their biological multi-person units that are often connected by propagation of biological codes or reproductive cells of individuals into others. While people do acknowledge the right to identify with ancestors and to have children, most societies acknowledge autonomy to the point that one can be free of an ancestor's criminal convictions and build one's own life where one can prove oneself, though available opportunities may in reality, in many cases depend on a person's past background that extend into a network of pre-existing connections. In extreme cases, one can also relinquish one's decedents from property rights.

While the proper dynamics of such relations can be debated and analysed fully, as well the existence of equal opportunities for individuals in a society that declares all legal entities to be equal citizens, in this paper, we simply acknowledge the existence of familial ties on an average and the human tendency to identify oneself with past entities as well as future ones besides one's own existence. The past and present entities may extend to the origin and propagation of one's physical existence. It is when searching for one's physical origin that one traces back one's biological ancestry and it is when extending one's existence to physical reproductions that one identifies with future rights of children and other close family.

\section{Genes and Information}

Through one's biological ancestors and descendent, one can be presumed to have extended one's biological origin and propagation. In strict technical terms, this concept is perplexing because in each generation, only fifty percent of a parent's DNA is propagated to the child. While this direct link is guaranteed, crossovers during meiosis mix up genetic material from grand-parents, and while within the fifty percent of material propagated, usually a fraction of each grand parent's genes do make it into a child's constitution, technically speaking, in extreme cases, very little or none of one grand parent's genes may be part of a child's genetic constitution. In reality too, some genetic material may be lost in the historical context even if all individuals are expected to leave behind progenies (genetic bottleneck).

However, at each node, where an offspring is born from a pair of parents, propagation of genetic material is guaranteed from each parent. While human genomes are 99.9 percent similar (within the same sex), within the human species, these slight differences contribute to a distribution of physical attributes and also in distinguishing looks. Early developmental experiences and hormones as well as genetic signals give rise to small differences within secondary structures of brain folds and creating individualized differentiation within functional connectivity in each human brain [20]. A parent can find his or her own bits and parts in the physical attributes or in some characteristics of a child, even though a child may be genetically completely distinct form one of its ancestors.
Yet one often traces back one's lineage to ancestors many generations back. This is a paradox given the number of cross breeding between any two separated nodes within a line makes it impossible to clarify how much of a person's genetic material is propagated. It is possible that none of an ancestor's genetic material is present in a descendant's gene pool.

Two points relevant to this paradox might be, the passing of knowledge and mores at each node, elaborated in the next paragraph, and also broad cultural characteristics within populations who might have shared overlapping ancestors or collaborators at some point. Also, even if an ancestor fails to push in any bit of his or her own genetic material into a descendant (of the opposite sex since the Y chromosome and mitochondrial DNA are propagated unchanged within the same sex), it is possible that bits of DNA have similar sequences even if they come from other people. The human genome is 99.9 percent alike, and broader characteristics might be conserved within unrelated people. People with same blood groups may have no relation and parents and progeny may have different blood groups (eg. A, B and $\mathrm{AB}$ ).

However, at each node, each parent has fifty percent overlap with a child's DNA and when the same biological parent brings up the child, the parent also environmentally trains the child with information and customs developed within the familial line alongside any formal education received within a broader community.

\section{Gene Enhancing and Improvement of Self}

The creation of designer babies by making use of gene editing technologies such as more improved versions of CRISPR in the future will change the genome of the child and not the parents. While it is possible that the genes edited will be small ones, keeping large degrees of semblances between parent and child genome, a very small mutation may enhance performance or differentiate a trait based on the expression of the product protein in other previously silenced portions of the gene or because of complex interactions (butterfly effect) in the same manner that some point mutations can also be lethal.

In an interesting case, a genetically deaf couple opted to have a baby made by donated reproductive cells who also would be dead [21]. The trait, which is genetic, formed such an important part of their personhood and what they wished to conserve within the family "culture."

If genes can be seen as blueprints for information about how to build the body which extends out to the world as a vehicle of conveying parts of an individual's personhood and also folds into internal functional units that give rise to internal parameters affecting the individual's health, wellbeing, mood and personality when combined with external factors such as foetal conditions, early childhood experiences, training and learning, then it is possible that genetically engineered babies will have traits that will be very different from parents' traits. Now many of an individual's traits are a combination of 
complex processes and even if parents share fifty percent of the DNA, interactions among gene segments from different parents and also the environment may produce traits that are very different from the parents' traits in a child by triggering and silencing certain exons. For example, very intelligent children may be born to people of normal intelligence and musical prodigies may be born to people who have no musical inclination.

Yet, it has been seen that when parents who are unable to conceive look for donated eggs or sperms, the attributes of the donors are considered carefully [14]. Often sperms or eggs are sought from very successful people. While not many very exceptionally successful people have been seen to produce offspring that are equally exceptional, the propensity to seek highly successful parent genomes indicate the inherent beliefs that some properties of that successful person will be conserved, hence one's belief in an individual perpetuating through his or her offspring.

As some of such artificially produced children have later sought their own biological parents or siblings [9], a largescale introduction of designer traits in a generation similarly evokes the possibility that such designer childrenseeking their designer siblings out, other children to whom such traits were artificially induced, and in the case of having unique traits introduced, feeling isolated and disconnected. In the case of donor eggs, the interest in seeking individuals with specific traits by recipient parents had led to some of the children searching for the same traits in the biological donors that had been procured by the recipient parents who lacked those traits. Such commodification[7] might lead to deterioration of valued in the case of designer children as well as donor eggs and sperms. It possibly remains a question to be answered whether designed children will feel similarly isolated from parents who bought in traits they did not have but coveted.

\section{THE SEXES AND IN BETWEEN}

In this second part of the paper, we extend concepts already discussed in the first part to understand the interdependence of the sexes and the possible limits of allowing one to perpetuate as one wishes using modern technologies.

While surely people with intersex attributes or desire for same sexes exist, a large majority of the human population identify with one of two sexes. Though at an individual level, human beings with rare qualities can come from any sex, on an average, although human beings are genetically 99.9 percent similar, that is only within the same sex. The Y chromosome is conserved from father to son and mainly codes for initiation of male sexual attributes. Some other functions such as specific immunological activities may also be attributed to the $\mathrm{Y}$ chromosome. On an average female immunity is different from the male immune system[18]. However,males and females also differ in how certain neurotransmitters such as serotonin are processed [24], making females more susceptible to depression. Typically, a male body is larger. That makes many of male organs such as liver, heart etc, larger on an average. The proportion of inter hemispheric connections in females is larger, though that might be a function of body weight[12]. The larger size of the male brain is also a function of body weight and brains of smaller males have been found to be smaller as well [12].Behavioural differences have been often associated with cultural upbringing [25]. These are grey areas where this paper has no point of view. However, male brains are also more prone to Parkinson's disease with higher propensity to lose dopaminergic neurons [3]. Furthermore, sex hormones have been linked with the functioning of certain other organs including the brain too, and in the creation of subtle differences in regulating gene networks[19] though discrepancy exist in the reporting of whether the male SRY gene is expressed in the brain or not[16].

Human abilities, though are an expression of many complex variables and hence, even if there might be average differences in different biological processes, a female may top a typically male role and vice versa. There had been accomplished queens and mathematicians whether or not these traits have any statistical link with success and gender.The effect of one variable may be compensated by others if any gender related correlation even existed. No conclusive investigations have been carried out. Similarly, in human exceptions also exist in humans who possess $\mathrm{XXX}, \mathrm{XXY}$ or XO chromosomes.

In this paper, we are mostly concerned with average biological tendencies that have biologically traditionally been part of human social behaviour and interconnectivity that might have played a role in the advancing society up until the modern age and in biological selection.

The author does not have any moral point of view regarding the exceptions or any view about whether any specific difference gives rise to any distinction in complex abilities as those issues are irrelevant here and are possibly not even well defined. Neither does the author have any point of view regarding the exceptions being the norm or not. Those debates have been carried out by many in literature.

A very relevant issue regarding the topic might be how males were more susceptible to COVID during the pandemic. Reference [4] shows how a combination of a biomolecular distinctiveness in certain subtle areas such as expression of ACE receptors, immunological differences based on chromosomal and hormonal attributes and life style are all involved.

It is almost impossible to disentangle these and assert that taking out one would equate the rest in a certain complex trait unless one variable is indeed completely removed from the equation. Given the result is unknown, the cost of removing one variable completely in a sudden burst is also possibly unknown. However, the author does maintain that social accumulation of knowledge and norms as well as individual genetics plays a role in the person's final expression of attributes. The social norms surely developed in many ways by larger needs of the society as well as because of existing 
differences in what one offered. Bargaining powers might have been correlated with weaknesses in areas unrelated with one specific variable over which dividing lines were drawn. The interplay of many variables entangled over complex histories make it difficult to ascertain what "would have" happened if a single trait such as body mass was removed and the author has no opinion just as the author has no opinion about if all the differences would have diminished if a single property was taken out. Rather, this paper is interested in conjecturing what broad changes might happen if certain practises were introduced in both extreme cases, when individuals would become otherwise exactly the same and when they would remain different to some extent.

In nature, life forms exist that are unisexual or bisexual morphologically. Sex differentiation can be traced back to at most 160 million years[26]. In some animals, the $\mathrm{Y}$ chromosome, which cannot undergo crossovers during meiosis has shrunk to the point that the attributes typically found in the $\mathrm{Y}$ chromosome either got reorganized and travelled to the $\mathrm{X}$ chromosomeas it did for some species of moles [1], or differentiated into $\mathrm{X}$ bearing females and $\mathrm{XY}$ bearing males as in some mice [5].

In species like humans, the $\mathrm{Y}$ chromosome is shorter than other chromosomes and does not undergo crossover. However, no loss of genes has been seen since chimpanzees evolved into humans [15]. The $\mathrm{Y}$ chromosome and the $\mathrm{X}$ chromosome respectively contain genes that are useful for the male and the female sexes and harmful to the opposite sexes, and this arrangement has been relatively stable in humans.

It is unclear how human society would have evolved had there been no sex differentiation or what will become of the human species if some day the $\mathrm{Y}$ chromosome becomes lethally mutated. However, as the arrangement stands from a strictly biological point of view, sexual reproduction involves meiosis and crossover within one's own cell before it becomes a reproductive cell and the next generation is created by combining genetic material from two cells. This allows for greater diversity and often compensates for recessive harmful genes. Surely something similar would have possibly been possible if only one sex existed within mankind and any two individuals within that sex could undergo similar sexual reproduction like warms and other hermaphrodites do. However, there are two sexes within the human species and one is unlikely to disappear soon.

As was mentioned above, despite the sure possibility of exceptions in any individual, physiological and hormonal differentiations exist between the sexes whether in subtle (brain connectivity) or gross forms (body weight). Even though with the advent of new technologies many reasons to have stratification of jobs based on physical properties have diminished in many cases, genetic dissimilarity between two members of the opposite sex remains more pronounced than geneticdissimilarities between members of the same sex. The Y chromosome and the SRY gene related factors also introduce obvious physiological broad differences. However, the genetic difference may or may not affect all parts of the body. Not all genes may be expressed at all times.

We imagine scenarios where:

1. Any individual can use his or her stem cells to have biologically similar reproductions.

2. Use his stem cells to produce either an egg or a sperm, in the case of males the sperm either containing the $\mathrm{X}$ or the $\mathrm{Y}$ chromosome and in the case of females, the sperm always containing the $\mathrm{X}$ chromosome. In such situation, any two members of the same sex will be able to reproduce artificially.

We try to surmise what extreme results might be from a strictly biological point of view.

The author would like to clarify that her belief and understanding is that the human social differential criteria are fairly stochastic. Hence, rare points and points not strictly adhering to expected properties are possible and are in many cases tolerated by a stable society. This paper is simply interested in understanding the effects of grossly shifting average behaviours from a biological and system dynamical point of view. We consider both the extreme scenarios when

1. Sexual reproduction allows for crossovers and also for creations of biological relations within individuals. While such mechanisms create clusters of some similarities, many attributes within that cluster remains dissimilar as even two siblings may differ in taste and talent vastly. The introduction of a different individual's chromosomes compensates for lethal or damaging individuals to some extent and also creates clusters where social responsibilities are shared, especially when rearing children that each can connect with biologically. Though not only chromosomes but individuals with different perspectives, values and possibly even cultures or heritages are introduced to create units, conflicts are possible within the unit, creating discontent or instability. A person given the technical ability to reproduce individually may be able to get rid of broad patches of "injustice" or conflicts often embedded in long term relations, where one party might even feel exploited. However, instead of creating localized clusters of similarities, the ability to produce one's own child would create even more focused similarity clusters that are narrower and concentrated on a single individual lines. This would also take away the forced imposition of disparate values that may smooth out some greater patches of conflicts by demanding degrees of compromise. However, even narrower and non-random clustering will not only reduce the effects of lethal or harmful mutations within a society, but will further isolate individuals as peaked clusters together with progeny that are very similar with further separations created with other members as generations pass. After many generations, the trusted dissemination of information within units of disparate 
will be reduced, and information will be more trapped within individual lines.

However, in a human society, large degrees of cooperation are needed as complex societies demand many different specializations of skills that are interconnected within larger scheme.

There seems to be a conflict between the society's interest in producing diverse random individuals with possible shuffling among genes that would create enhanced abilities for survival of that individual and for the cluster, and one's own interest in propagating oneself throughout generations. However, when the balance between the two is broken, either a person may find the act of rearing children to be a burden and view it as work done for others, or individuals may continue as more stable lines with fewer changes, clustering interests and information into focused self-centred groups that also affect the cohesive factor introduced by commonalities generations back within a larger population besides exposing one to his or her own weakness vastly by accentuating damaging mutations.

2. Reference [2] discusses a scenario where a single person uses his own stem cells to grow both an egg and a sperm to have a child. We consider a slightly different scenario.

If each individual can be technically enhanced with the ability to produce either an egg or a sperm by the use of stem cell technology, males shall still have the choice to reproduce to create a male or a female, but a female producing a sperm will only be able to produce a female.

In that case, males clustered together will be able to recreate the current composition of thehuman society but females clustered together will only be able to create a society of females.

If in the larger society, only a section decides to use this technology, then there will simply be one or more subclusters capable of propagating completely excluding the other section.

Now, as human society is stochastic, even in the absence of such technologies, children created by a mother and a father may be reared by a single sex or a single parent. However, the child can trace back its origin to two sexes biologically.

In an artificially created single sex cluster, the larger autosomal content may be shared by members of the other sex through distant relatives before any such division. However, it is to be noted that human beings are largely similar while the single Y chromosome creates a much larger genetic variation, which, within a traditional human cluster, is related directly with members of other sexes within units that reproduce together.

If direct biological connections are broken down between these clusters, they will remain somewhat separated within a larger pool or in isolated forms, divided by gross average differences in traits mostly related to gender specific attributes due to less than one hundred protein coding genes in the $\mathrm{Y}$ chromosome [27]. If the clusters remain isolated for generations, these differences may add to mutations giving rise to drifts and hence make the differences even more pronounced.

If the two sexes possess certain traits that are on an average complimentary, such distancing within genetic and cultural/information pools may lead to the clusters treating each other in manners similar to different species since the separation between the sex chromosomes is larger than the difference between genomes of two individuals within the same sex and they will be reproducing within their own cluster. But the complementary needs will be in demand and so use of complimentary traits without the existence of any cohesive familial ties will be sought. Even if these subclusters are parts of a larger society where both are citizens in a less extreme case, such interests may lead to high levels of exploitation as is observed among different isolated classes among which no reproduction of genetic shuffling takes place.

On the other hand, if the two clusters are indeed statistically exactly the same except for these attributes that are only reflected in reproductive behaviours or history, which become obsolete, the two clusters will become like two populations within the human species that have very similar abilities or potentials but that are separated by gross differences in looks and physical strength in the cluster that is solely constituted of females derived from females given males cannot be derived from them.

While it is unclear how child rearing activities will be carried out in a cluster derived only from males that contains both males and females, in the female only cluster, the members will have to provide for both child rearing and defence and in the few remaining areas where physical strength might still be of importance. Otherwise, one specific cluster will be dependent on the other only for physical strength related services while remaining self-sufficient in other areas.

However, the role of hormones in psychology has not been yet biologically refuted [19]. Hence, the two subclusters might yet have separations based on psychological and disease related vulnerabilities correlated with male or female looks that allows for further categorization and hence acting as barriers in bonding based on similarity if all other complementary needs can be satisfied within an individual cluster when the large number of data points within a cluster allows for it.

\section{A.Exploitations and Domestic Laws}

Given the large number of variables in the evolution of human civilisations and the impossibility disentangling the contributions of many subtle components in a complex system that expressed as a whole containing sexes and also individuals - both factors giving rise to subtle differences and fluctuating traits that are often not easy to categorize strictly based on a single variable, it is impossible to predict with certainly what the cost of completely shifting naturally 
evolved average human behaviour by using artificial means will be in a delicately balanced system that depends on accumulated knowledge, trust and loyalty in a complicated manner.

An argument about having the sexes separated is exploitation of the weaker sex, which in most cases, is the female that is physically weaker. If this argument leads to further segregation of the sexes, we may imagine having clusters of males and females again with the same issues but with the weaknesses concentrated within subclusters. While many modern work opportunities do not require physical strength and many jobs have shifting gender balance too, such a separation will also separate the reproductive attributes that are dictated by hormones and not just by the possibilities of having stem cells produced from stem cells. In a segregated society, the interdependence between the sexes will be lower, and two factions will eventually arise, one with significantly lower strength and some anxiety, mood related differences based on neurotransmitter and immune response related attributes even if no other such differences exist. The subclusters will not have a right over each other because of any pre assumed physical or mental complimentary attributes or shared sense of unity other than shared resources or citizenship which allows individuals to have rights. Hence, they will not owe each other any extra favours because of interdependence between their traits.

This is a complex scenario with many grey areas yet unproven or partially proven to predict whether one cluster will try to colonize the other or if diversity rights will protect any individual irrespective of any cluster membership simply because the society cannot dispense any of the clusters. However, again, if the traits of both the clusters are exactly the same, except for a difference in physical strength, in blue collar jobs, this specific attribute still may play a role as well as in direct combat situations in a world that still has armed conflicts. Physical strength may be a factor alsoin certain areas of criminal activities. It is easy to imagine that in such a scenario, criminals from the physically strong cluster might target the weaker cluster that is emotionally and geographically detached.

Again, even if two clusters become independent, or if sex becomes redundant, weaker members of the same sex may still be exploited by the stronger ones because of domestic and personal needs even if professional and public areas are more strictly controlled. In [19], it was shown that even within the same sex, fluctuations of hormone levels between individuals may trigger the activation ofseparate gene networks among individuals of the same sex group, hence producing individuals that are not similar in behaviour based on how they were exposed to hormones. Exploitations of individuals of such differentiating traits by the opposite types within the same sex may not be ruled out either.

On the other hand, if a largely traditional setting with both the sexes inside reproduction units is imagined, the author believes that a modification of domestic abuse laws to include laws that would prohibit a partner from allowing the other from gaining financial independence based on whatever talent or skill he or she might possess and a larger scale education that values skills across the spectra of traditionally held male and female traits whether or not there are statistical difference in final job distribution in any specific areas, might enable each individual be respected for his or her worth for the ability he or she actually might be able to prove without assuming either equal or categorical statistical distributions.

\section{CONCLUSION}

Many human forms of behaviour manifest within the human society as stochastic variables. A society can tolerate deviations from the average and might even be enhanced by such deviations at least to some extent in many cases. However, in this paper, we investigated the values held in the society regarding one's biological origins and how modern technologies such as gene editing or stem cell technology can affect the person's sense of origin as well as change the traditional balances within a society. Modern technology has also changed the work scene in many areas. However, the human society remains a complex network of individuals tied together with genetic and emotional connections and with loyalty towards others within the same cluster derived from biological as well as artificially created factors. A drastic change introduced artificially in such a delicate system may come with unforeseen costs that were imagined in the abovementioned scenarios. A greater care in updated laws that protect individuals from exploitations within the cluster to adapt with modern needs may be considered while taking a more cautious step in introducing technologies that allow for large scale artificially induced shifts in biologically held balances.

\section{REFERENCES}

[1] Arakawa,Y,C. Nishida-Umehara C.,MatsudaY,,Sutou S., Suzuki H., (2002). X-chromosomal localization of mammalian Y-linked genes in two XO species of the Ryukyu spiny rat. Cytogenetic and Genome $\quad$ Research. $\quad 99$ (1-4): 303-9. doi:10.1159/000071608.PMID 12900579. S2CID 39633026.

[2] Bowman-Smart, H., (2021). Orphans by Design,: The Future of Genetic Parenthood.Bioethics. 35: 23-30.

[3] Buck,Silus A.,(2021). Vesicular glutamate transporter modulates sex differences in dopamine neuron-vulnerability to age-related neurodegeneration. Aging Cell. 20(5).

[4] Bwire, GM.,(2020). Coronavirus: Why Men are More Vulnerable to Covid-19 Than Women? [published online ahead of print, 2020 Jun 4]. SN Compr Clin Med. 1-3. doi:10.1007/s42399-020-00341w

[5] Charlesworth, B, Dempsey N D.,. (April 2001). A model of the evolution of the unusual sex chromosome system of Microtus oregoni. Heredity. 86 (Pt 4): 387-94.doi:10.1046/j.13652540.2001.00803.x. PMID 11520338. S2CID 34489270.

[6] Chmielewski, Wendy E.,(2001). Review of Desire and Duty at Oneida: Tirzah Miller's Intimate Memoir. Utopian Studies. 12 (1): 176-178. ISBN 9780815621690. JSTOR 20718260.

[7] Cohen, IG.,(2017). Sperm and Egg Donor Anonymity: Legal and Ethical Issues. The Oxford Handbook of Reproductive Ethics.

[8] Crow, J.,(2002). Unequal by nature: a geneticist's perspective on human differences. Daedalus. 131(1):81-88.

[9] Dominus, S.,.(2019). Sperm Donors Cannot Remain Anonymous Anymore. Here's What that Means. New York Times Magazine. 
https://www.nytimes.com/2019/06/26/magazine/sperm-donorquestions.html.

[10] Douglas, T., and Devolder, K.,(2019). A conception of genetic parenthood. Bioethics. 33(1): 54-59.

[11] Dworkin,R.,.(2003). Equality, Luck and Hierarchy. Philosophy and Public Affairs. 31: 190.

[12] Eliot, L.,Ahmed A.,, Khan H., Patel J.,(2021). Dump the 'dimorphism': Comprehensive synthesis of human brain studies reveals few male-female differences beyond size. Neuroscience \&Biobehavioral Reviews. 125: 667 DOI: 10.1016/j.neubiorev.2021.02.026

[13] Ellis, N.,(Oct 19, 2021). Lost Lineage: The Quest to Identify Black American' Roots. The Washington Post. https://www.washingtonpost.com/nation/2020/02/25/lost-lineagequest-identify-black-americans-roots/

[14] Epstein, Sonia F. and Whitehouse P. N.,(April 30, 2020). Inheriting the Ivy League: The Market for Educated Egg and Sperm Donors. The Harvard Crimson.

[15] Fischman, J.,(April 23, 2014). Reprieve for Men: Y chromosome is not vanishing. Scientific American. The Human Protein Atlas. https://www.proteinatlas.org/ENSG00000184895-SRY/tissue. Accessed Oct 24, 2021.

[16] Hegel, G. W. F. and Dayide S. W.,(1896). Hegel's Philosophy of right. London: G. Bell.

[17] Klein, S. and FlanaganK...(2016). Sex differences in immune responses. Nat Rev Immuno.l 16,626-638. https://doi.org/10.1038/nri.2016.90.
[18] Marrocco, J. andMcEwen B. S. (2016). Sex in the brain: hormones and sex differences. Dialogues in clinical neuroscience.

[19] Mueller, S., Wang D.,Fox, M. D., Michael, B. T.,YeoT.,Sepulcre J.,Sabuncu, M. R.., Shafee R.,, Lu, J.,Hesheng.L.,(2013). Individual Variability in Functional Connectivity Architecture of the Human Brain. Neuron. Volume 77, Issue 3: 586-595, https://doi.org/10.1016/j.neuron.2012.12.028.

[20] Mundy, L.(March 31 2002). A world of their own. The Washington Post. Accessed April 62002 at http://www.washingtonpost.com/wp-dyn/articles/A231942002Mar27.html

[21] Plato. (1943). Plato's The Republic.New York :Books, Inc.

[22] Pymont K.,(2014). Thinking of excluding children from your inheritance? It may be harder than you think, Kingsley Napley Dispute Resolution Law Blog, 17 July 2014, https://www.kingsleynapley.co.uk/insights/blogs/disputeresolution-law-blog/thinking-of-excluding-children-from-yourinheritance-it-may-be-harder-than-you-think

[23] Rehbein, E, Hornung J., SundströmPoromaa I.,Dernt B.,(2021). Shaping of the Female Human Brain by Sex Hormones: A Review.Neuroendocrinology. 111:183-206. doi: 10.1159/000507083.

[24] Rippon, G. (2019). The Gendered Brain, Random House

[25] Warren, WC.,Hillier,LW. et al., (2008).Genome analysis of the platypus reveals unique signatures of evolution. Nature. 453 (7192): 175-83.

[26] Williams, S. CP.,(2014). Y chromosome is more than a sex switch, Science, 23 April, 2014 